\title{
Estudo da Dispersão de Argilas Organofílicas em Nanocompósitos de Prolipropileno Obtidos pelo Método em Solução com Auxílio de Ultrassom
}

\author{
Eveline Bischoff, Kelly Silva dos Santos, Susana Alcira Liberman, Raquel Santos Mauler \\ Instituto de Química, Departamento de Química Orgânica, UFRGS
}

\begin{abstract}
Resumo: Nanocompósitos de polipropileno PP/OMMT foram preparados com o uso de duas argilas modificadas organofilicamente (C-15A e I-44P) e de um compatibilizante (PP-g-MA) pelo método em solução utilizando-se um banho de ultrassom. O objetivo deste estudo foi compreender a morfologia dos nanocompósitos, ou seja, o grau de dispersão destas argilas na matriz de polipropileno, e consequentemente, correlacioná-la com as propriedades finais obtidas. A morfologia dos nanocompósitos foi avaliada por Difração de Raios X (DRX) e Microscopia Eletrônica de Transmissão (MET). As propriedades mecânico-dinâmicas e térmicas dos nanocompósitos foram avaliadas por Análise Mecânico-Dinâmica (DMA) e Calorimetria Diferencial de Varredura (DSC). Análises estatísticas das imagens de transmissão foram realizadas possibilitando a obtenção da razão de aspecto (comprimento/espessura) das partículas de argila.
\end{abstract}

Palavras-chave: Argilas organofílicas, morfologia, nanocompósitos de PP, razão de aspecto, ultrassom.

\section{Dispersion of Organoclays in Polypropylene Nanocomposites by Ultrasound-assisted Solution Method}

\begin{abstract}
Polypropylene nanocomposites PP/OMMT were prepared using two modified organic clays $(\mathrm{C}-15 \mathrm{~A}$ and I-44P) and (PP-g-MA) as compatibilizer in the solution method using an ultrasound bath. The objective of this study was to understand the morphology of the nanocomposites and degree of clay dispersion in the polypropylene matrix, which are then correlated with the final properties. The morphology of nanocomposites was evaluated by X-ray Diffraction (XRD) and Transmission Electron Microscopy (TEM). The dynamic mechanical properties and thermal properties were measured by Dynamic Mechanical Analysis (DMA) and Differential Scanning Calorimetry (DSC). Statistical analyses of the transmission images were made in order to obtain the aspect ratio (length/thickness) of the clay particles.
\end{abstract}

Keywords: Organoclays, morphology, PP nanocomposites, aspect ratio, ultrasound.

\section{Introdução}

O polipropileno (PP) é um dos polímeros mais utilizados na indústria devido a sua alta versatilidade em termos de propriedades como: facilidade de processamento, alta resistência química, baixo custo, além de ser reciclável ${ }^{[1]}$. No entanto, para determinadas aplicações torna-se necessário o incremento de algumas propriedades como: estabilidade dimensional, resistência ao impacto e rigidez. Em virtude disso, a adição de cargas minerais em nível nanométrico tem sido empregada como método para se alcançar estes ganhos em propriedades ${ }^{[2]}$.

Dentre as nanocargas mais utilizadas na obtenção de nanocompósitos, a montmorilonita $(\mathrm{MMT})^{[3]}$ tem se destacado por apresentar estrutura em multicamadas e elevada razão de $\operatorname{aspecto~}^{[4]}$. Porém, a dispersão de nanocargas em poliolefinas não é favorecida termodinamicamente, visto que a afinidade química entre a MMT (carga inorgânica polar) e o PP (matriz orgânica apolar) é consideravelmente baixa. Para promover uma melhor compatibilidade é necessário modificar quimicamente a MMT tornando-a organofílica $(\mathrm{OMMT})^{[5]}$, processo que ocorre pela troca dos cátions sódio presentes nas galerias da argila por sais de amônio quaternário $^{[6]}$. O uso de agentes compatibilizantes ${ }^{[7]}$ é outra forma de promover uma melhor adesão interfacial entre carga e matriz e o polipropileno graftizado com anidrido maleico é um dos mais utilizados ${ }^{[1]}$.

Outro fator decisivo é o processo de obtenção dos nanocompósitos ${ }^{[8]}$, o qual influenciará no grau de dispersão das lâminas do argilomineral na matriz de PP. Um dos métodos mais utilizados para obtenção de nanocompósitos de PP é a intercalação por fusão ${ }^{[9]}$, no qual a mistura da argila com o polímero ocorre no estado fundido, mas uma dispersão em escala nanométrica muitas vezes não é obtida. No método em solução é possível o inchamento prévio da argila em um solvente com a finalidade de aumentar a distância entre suas camadas e, em seguida, dissolver o polímero. Este processo facilita a penetração das cadeias do polímero entre as camadas da OMMT ${ }^{[10]}$ e consequentemente, se obtém uma maior esfoliação da argila na matriz de PP. Esta metodologia permite compreender e correlacionar de forma mais eficaz a morfologia e as propriedades finais dos nanocompósitos apesar de, no caso de poliolefinas,

Autor para correspondência: Eveline Bischoff, Instituto de Química, Universidade Federal do Rio Grande do Sul - UFRGS, 
não ser um processo de interesse industrial. Além disso, pode-se fazer uso de dispositivos mecânicos que auxiliam na esfoliação como o processo de sonicação ${ }^{[11]}$.

O objetivo deste trabalho foi estudar a morfologia dos nanocompósitos obtidos pelo método em solução com auxílio de ultrassom, e sua influencia nas propriedades térmicas e mecânicas, utilizando diferentes concentrações de duas argilas organofílicas com diferentes quantidades de modificador orgânico e agente compatibilizante.

\section{Procedimento Experimental}

\section{Materiais}

Os materiais usados na preparaçãodosnanocompósitos foram PP homopolímero (IF $\left.\left(230^{\circ} \mathrm{C} / 2.16 \mathrm{~kg}\right): 3,5 \mathrm{~g} / 10 \mathrm{~min}\right)$ da Braskem S.A; argila montmorilonita sódica (MMT): Cloisite $\mathrm{Na}^{+}(\mathrm{MMT} \mathrm{Na})$ da Southern Clay Products; duas argilas montmorilonita organofílicas (OMMT) modificadas com sal de amônio quaternário contendo duas metilas e duas cadeias alquílicas longas hidrogenadas ligadas as átomo de nitrogênio e com distribuição aproximada de $65 \% \mathrm{C}_{18}, 30 \% \mathrm{C}_{16}$ e $5 \% \mathrm{C}_{14}$ : Cloisite $15 \mathrm{~A}$ (C-15A) da Southern Clay Products e Nanomer I-44P da Nanocor; PP funcionalizado com anidrido maleico (PPg-MA - Polybond 3002) (IF $\left(230^{\circ} \mathrm{C} / 2.16 \mathrm{~kg}\right.$ : $7 \mathrm{~g} / 10 \mathrm{~min}$ com $0.2 \% \mathrm{p} / \mathrm{p}$ anidrido maleico, da Chemtura; xileno P.A (densidade: $0,86 \mathrm{~g} \cdot \mathrm{cm}^{-3}$ ).

\section{Preparação dos nanocompósitos}

Os nanocompósitos de PP foram preparados pelo método de intercalação em solução utilizando xileno como solvente com 1 e $5 \% \mathrm{p} / \mathrm{p}$ de argila I-44P ou C-15A. Nas amostras nas quais foi utilizado agente compatibilizante, o seu teor foi o mesmo que o da argila (1:1). As composições das amostras podem ser visualizadas na Tabela 1. As amostras foram preparadas em duas etapas. Primeiramente, foi adicionado xileno na mistura dos componentes $(\mathrm{OMMT}+\mathrm{PP}-\mathrm{g}-\mathrm{MA}+\mathrm{PP})$ para promover, através do inchamento da argila, um aumento das distâncias interlamelares. Com o intuito de tornar ainda mais eficiente o processo de dispersão das cargas, foi utilizado um banho de ultrassom (Unique USC, modelo 1400) com frequência de $40 \mathrm{KHz}$ e potência de $135 \mathrm{~W}$, onde as amostras ficaram por um período de 4 horas, sob agitação mecânica a uma temperatura de $60^{\circ} \mathrm{C}$. Na segunda etapa de preparação, as amostras foram colocadas em uma chapa de aquecimento, sob agitação magnética, a uma temperatura de $130^{\circ} \mathrm{C}$ para que ocorresse a solubilização do polímero. Posteriormente, o solvente foi evaporado e todas as amostras foram secas à vácuo em uma estufa a $100^{\circ} \mathrm{C}$ para garantir a completa evaporação do solvente. Os corpos de prova para realização dos ensaios mecânico-dinâmicos e microscopia eletrônica de transmissão foram preparados por injeção em uma mini-injetora (Haake minijet II) com temperatura do cilindro a $190^{\circ} \mathrm{C}$ e do molde à temperatura ambiente. Para os demais ensaios (Raios-X e DSC) o material foi analisado na forma de filmes obtidos em prensa hidráulica (Carver, série Monarch, modelo 3710) a $190^{\circ} \mathrm{C}$.

\section{Caracterização dos nanocompósitos}

O teor de material orgânico das argilas foi obtido por termogravimetria em um TGA da TA model QA-50. As amostras, em forma de pó e sem nenhum tratamento prévio, foram aquecidas de $80^{\circ} \mathrm{C}$ até $900^{\circ} \mathrm{C}$ a uma taxa de $20^{\circ} \mathrm{C} / \mathrm{min}$ sob atmosfera de nitrogênio. Análises de Difração de Raios X (DRX) foram realizadas nas argilas, em forma de pó, para a obtenção das distâncias interlamelares.

A morfologia dos nanocompósitos de PP foi avaliada através das técnicas de DRX e Microscopia Eletrônica de Transmissão (MET). Todas as medidas de DRX foram realizadas em um difratômetro da marca Siemens D500 equipado com radiação $\mathrm{Cu} \mathrm{K} \alpha$ e operando com um comprimento de onda de $0.154 \mathrm{~nm}$. Os difratogramas foram obtidos com taxa de $0.05^{\circ} \mathrm{s}^{-1}$. As imagens de transmissão foram realizadas em um microscópio do tipo JEOL JEM-1200 Ex II, com tensão de aceleração de 80 kV. Para a obtenção dos cortes dos nanocompósitos foi utilizada uma câmara criogênica mantida na temperatura de $-80^{\circ} \mathrm{C}$, acoplada ao ultramicrótomo RMC CXL. Utilizou-se uma lâmina de vidro para polir a superfície da amostra e, em seguida, com o auxílio de uma lâmina de diamante, obtiveram-se cortes ultrafinos com espessura de aproximadamente $70 \mathrm{~nm}$, que foram coletados em telas de cobre de 300 mesh. A quantificação e determinação da razão de aspecto das partículas de OMMT foram obtidas através da análise das imagens de transmissão em ampliações de 30.000 vezes, utilizando o programa Image Tool.

As propriedades térmicas dos nanocompósitos de PP foram avaliadas através de Calorimetria Diferencial por Varredura (DSC) em um equipamento Thermal Analyst 2100 (TA Instruments) sob atmosfera de nitrogênio. As amostras foram aquecidas de $50^{\circ} \mathrm{C}$ até $200^{\circ} \mathrm{C}$ (isoterma

Tabela 1. Razão de aspecto dos nanocompósitos de PP/I44P e PP/C-15A

\begin{tabular}{ccccc}
\hline Amostras & $\mathbf{N}^{\circ}$ total de partículas & Comprimento médio $(\mu \mathbf{m})$ & Espessura média $(\mu \mathbf{m})$ & Razão de aspecto \\
\hline 1\% I44P + PP & 23 & 0,33 & 0.042 & 7,8 \\
$1 \%$ I44P + 1\% PP-g-MA + PP & 41 & 0,32 & 0,035 & 9,1 \\
$5 \%$ I44P + PP & 54 & 0,26 & 0,033 & 8,1 \\
$5 \%$ I44P + 5\% PP-g-MA + PP & 181 & 0,27 & 0,022 & 12,2 \\
1\% C-15A + PP & 63 & 0.29 & 0.045 & 6.4 \\
$1 \%$ C-15A + 1\% PP-g-MA & 35 & 0.26 & 0.038 & 6,8 \\
5\% C-15A + PP & 119 & 0.35 & 0.031 & 11,2 \\
$5 \%$ C-15A + 5\% PP-g-MA & 239 & 0.21 & 0.021 & 10,0 \\
\hline
\end{tabular}


por 5 minutos) com uma taxa de aquecimento e resfriamento de $10^{\circ} \mathrm{C} / \mathrm{min}$. $\mathrm{O}$ grau de cristalinidade foi determinado utilizando-se $\Delta \mathrm{H}_{\mathrm{m}}{ }^{0}=190 \mathrm{~J} / \mathrm{g}$ para o $\mathrm{PP}^{[12]}$. As propriedades mecânico-dinâmicas (DMA) foram realizadas em um equipamento TA instruments Q-800 no modo "single cantilever" a $1 \mathrm{~Hz}$. As amostras injetadas foram aquecidas de -30 (isoterma por 10 minutos) até $130^{\circ} \mathrm{C}$ a uma taxa de aquecimento de $3^{\circ} \mathrm{C} / \mathrm{min}$.

\section{Resultados e Discussão}

\section{Caracterização das argilas}

A distância interlamelar é característica de cada argila e esta distância varia principalmente com a quantidade e estrutura do cátion orgânico presente entre as camadas ${ }^{[13]}$. Na Tabela 2 encontram-se os valores dos ângulos de difração $2 \theta$ e das distâncias intelamelares $\left(\mathrm{d}_{001}\right)$ obtidas através da lei de Bragg, assim como a quantidade de teor orgânico presente nas argilas organofílicas utilizadas.

A modificação orgânica possibilitou a estas argilas um aumento na distância interlamelar quando comparadas a argila MMT sódica (MMT Na), este aumento foi de 1,28 para 3,4 e 2,9 para as argilas C-15A e I-44P, respectivamente. As argilas C-15A e I-44P contém o mesmo tipo de modificador orgânico, no entanto, devido a maior quantidade presente na argila C-15A acaba resultando num aumento de $17 \%$ na distância interlamelar. Em trabalho anterior, já foi demonstrado que o uso ultrassom é capaz de quebrar as lamelas da argila, reduzindo sua razão de aspecto de 500nm para $300 \mathrm{~nm}$ quando comparado ao mesmo sistema utilizando apenas solvente ${ }^{[14]}$.

\section{Difração raios $X$}

A avaliação dos nanocompósitos por difração de Raios X é um indicativo da presença de estruturas intercaladas/esfoliadas. Na Figura 1 são mostrados os difratogramas dos nanocompósitos com as argilas C-15A e I-44P e pode ser observado um leve deslocamento para ângulos menores nos nanocompósitos quando comparados aos das argilas puras, confirmando aumento na distância interlamelar.

Para o nanocompósito com 5\% de C-15A, verifica-se que não ocorreu deslocamento do pico com relação ao da argila pura. Apesar disso, pela análise das micrografias de transmissão, que serão discutidas posteriormente, foi possível visualizar lamelas individuais de argila na matriz polimérica. Para as demais amostras, o deslocamento dos picos para ângulos menores evidencia o efeito do solvente

Tabela 2. Cálculo das distâncias interlamelares e dos resíduos orgânicos das argilas organofílicas.

\begin{tabular}{cccc}
\hline Argila & $\mathbf{2} \theta\left(^{\circ}\right)$ & $\mathbf{d}_{\mathbf{0 0 1}}(\mathbf{n m})$ & $\begin{array}{c}\text { Teor de material orgânico* } \\
(\mathbf{\%} \text { em peso) }\end{array}$ \\
\hline MMT Na & 6,9 & 1,28 & - \\
C-15A & 2,6 & 3,4 & 42,2 \\
I44P & 3,0 & 2,9 & 37,0 \\
\hline
\end{tabular}

*Modificador $\left(\mathbf{C H}_{3}\right)_{2}(\mathbf{H T})_{2} \mathbf{N}^{+}-\mathrm{HT}=$ grupamento graxo hidrogenado $\left(\cong 65 \% \mathrm{C}_{18}, 30 \% \mathrm{C}_{16} \cong 5 \% \mathrm{C}_{14}\right)$ e contra íon $=\mathrm{Cl}^{-}$. que ao provocar o inchamento da argila acaba favorecendo o deslizamento das lâminas e, consequentemente, a esfoliação.

Para os nanocompósitos com a I-44P, a distância interlamelar aumentou $21 \%$ enquanto que para aqueles com a C-15A somente $4 \%$. Esses resultados podem ser atribuídos à existência de um limite de expansão, devido ao grau de modificação das argilas. Atingido esse limite não ocorre mais variação na distância interlamelar até iniciar o processo de esfoliação. Como a C-15A é mais modificada acaba tendo um deslocamento menor das lâminas pelo efeito do solvente.

$\mathrm{O}$ uso de PP-g-MA deslocou os picos para ângulos um pouco menores, entretanto o seu efeito ficou mais evidente nos nanocompósitos com a I-44P independente da concentração. Os picos observados sugerem ainda a presença de estruturas ordenadas, do tipo tactóides em ambas as argilas, indicando que não houve esfoliação total mesmo com o processo de preparação com solvente e ultrassom.

A intensidade dos picos aumentou com o aumento da quantidade de argila empregada como já era esperado. Os nanocompósitos com I-44P e compatibilizante apresentaram menor intensidade quando comparados aos

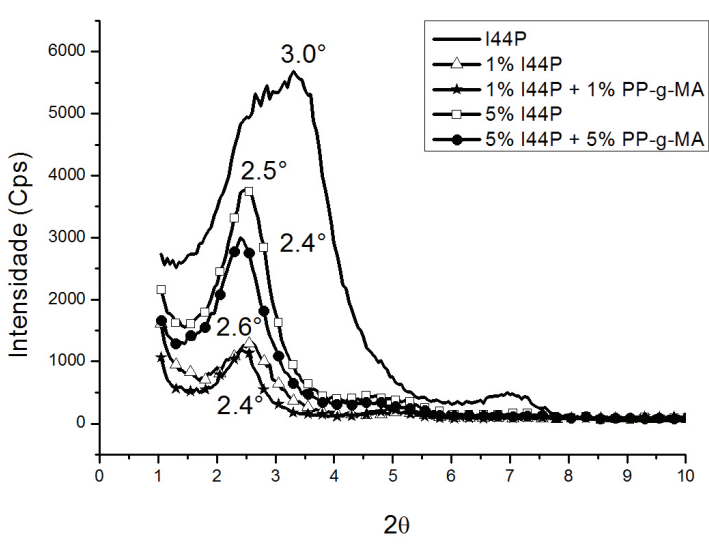

(a)

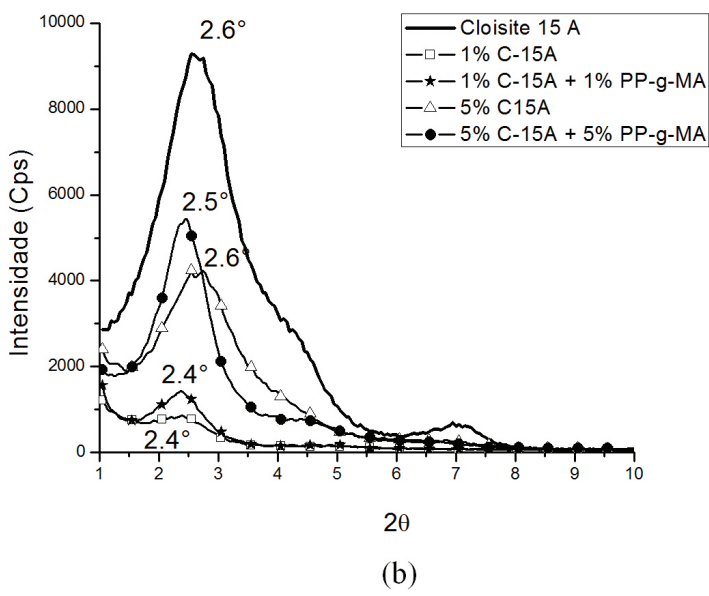

Figura 1. Difratogramas dos nanocompósitos: (a) PP/I44P e (b) PP/C-15A. 
sem PP-g-MA, independente de sua concentração. Esse decréscimo da intensidade dos picos pode ser atribuído à quebra dos aglomerados ocasionando maior dispersão das lamelas da argila ao longo da matriz ${ }^{[5,15]}$. Hotta e $\mathrm{Paul}^{[16]}$ mostraram que para misturas de PELBD com argilas modificadas com $\left(\mathrm{CH}_{3}\right)_{2}(\mathrm{HT})_{2} \mathrm{~N}^{+}$mesmo para altas concentrações de compatibilizante em relação a argila, o deslocamento do pico foi similar para todos os nanocompósitos apenas diminuindo a intensidade com o aumento do teor de compatibilizante.

\section{Morfologia dos nanocompósitos de PP/OMMT}

As imagens de MET dos nanocompósitos de PP (Figuras 2 e 3) mostraram a presença de alguns aglomerados, além de lamelas esfoliadas (lâminas de espessura menor que $10 \mathrm{~nm}$ ) e bem distribuídos ao longo da matriz, principalmente para as amostras com a C-15A. Santos et al. demonstraram que a maior quantidade de sal de amônio presente entre as folhas da C-15A proporciona uma melhor orientação e uma menor flexibilidade das partículas esfoliadas, e que o uso de ultrasom melhora a distribuição das lamelas da argila ${ }^{[14]}$. Como essa argila possui uma maior distância entre as suas camadas, há uma menor força de interação entre elas, permitindo uma separação mais rápida e eficaz estimulada pelo processo de sonicação. Para a argila I44P, o processo de separação é mais lento em razão da menor distância entre suas camadas, o que diminui a eficiência da sonicação ${ }^{[14]}$.

$\mathrm{Na}$ maior concentração de OMMT $(5 \% \mathrm{p} / \mathrm{p})$, a dispersão e distribuição das partículas se mantiveram proporcionais, ou seja, quanto maior a concentração de argila maior foi o número de partículas dispersas homogeneamente na matriz de PP.

A presença do agente compatibilizante melhorou a dispersão e distribuição das partículas de argila quando comparadas ao mesmo sistema sem PP-g-MA. Esse efeito ficou mais evidente para os sistemas com a I-44P, devido a esta argila apresentar menor quantidade de sal de amônio, permitindo assim, uma maior interação entre os grupos polares do PP-g-MA e os grupos hidroxila $(-\mathrm{OH})$ presentes na superfície da OMMT. Estes resultados estão de acordo com aqueles observados na difração de raios X.

O grau de dispersão das partículas de argila na matriz polimérica está diretamente relacionado com a sua capacidade de delaminação o que, consequentemente, determina a sua razão de aspecto (comprimento/ espessura $)^{[17]}$ e a eficiência do reforço na matriz ${ }^{[18]}$. $\mathrm{O}$ comprimento e espessura das partículas foram determinados nos nanocompósitos com e sem PP-g-MA através de analises estatísticas das imagens de MET em uma área total de 19,8 $\mu^{2}$. Na Figura 4 são mostrados dois exemplos de histogramas que representam a distribuição de comprimentos e espessuras para as amostras com 5\% C-15A com e sem compatibilizante. O resultado das determinações da razão de aspecto das argilas mostra a complexidade da morfologia dos nanocompósitos. King et al. ${ }^{[19]}$ estudaram através de dispersão de raios X, dispersão de nêutrons a pequeno ângulo (SANS), e dispersão de nêutrons a ultrapequenos ângulos (USANS) o efeito do inchamento de argilas organofilicas em diferentes solventes e apresentaram um modelo estrutural para a argila parcialmente dispersa. Esse modelo consiste de tactóides aleatoriamente orientados com folhas de argila que se sobrepõem parcialmente e assim, as dimensões podem variar consideravelmente, afetando a razão de aspecto. A larga distribuição de tamanho das particulas que se observam nos nanocompósitos obtidos com auxilio de solventes também foi observada em nanocompósitos de polipropileno obtidos pelo processo de extrusão ${ }^{[15]}$.

Em virtude dos nanocompósitos terem sido preparados em solução, se observou uma maior delaminação das partículas de argila, o que está de acordo com resultados anteriores de Burgentzlé et al. ${ }^{[10]}$. As micrografias nas Figuras 2 e 3 mostram que muitas partículas se apresentam curvadas evidenciando sua flexibilidade. Esse aspecto dificulta a análise dimensional dessas estruturas, mas permite estabelecer as tendências morfológicas dos nanocompósitos

Na Tabela 1 são apresentados os valores da razão de aspecto e os correspondentes valores médios ponderais de comprimentos e espessuras medidos para o número de partículas indicado para cada uma das amostras. Com o aumento da concentração de argila, verificou-se a presença de um maior número de partículas, como esperado, já que o nível de dispersão se mantém elevado para altos teores de argila. Para os nanocompósitos com compatibilizante, o número de partículas foi superior quando comparado com o mesmo sistema sem compatibilizante, mas para a amostra com $1 \%$ de C-15A, a amostra com compatibilizante mostrou menos partículas, o que pode ser atribuído à heterogeneidade da amostra relacionada às pequenas áreas utilizadas para análises de microscopia eletrônica de transmissão.

Comparando os nanocompósitos com $1 \%$ e $5 \%$ de I-44P, se observa que para maiores teores de argila ocorreu diminuição do comprimento (de $0,33 \mu \mathrm{m}$ para $0,26 \mu \mathrm{m}$ ) e da espessura (de $0,042 \mu \mathrm{m}$ para $0,033 \mu \mathrm{m}$ ), e portanto, não houve variação significativa no valor da razão de aspecto. A presença do compatibilizante promoveu uma diminuição na espessura das partículas de argila, o que consequentemente, ocasionou uma maior razão de aspecto, comportamento já observado por Lee et al. ${ }^{[17,20]}$ em blendas de nanocompósitos de PP com elastômero utilizando PP-g-MA como compatibilizante.

Para a argila C-15A ocorreu um aumento no comprimento médio das partículas de 0,29 $\mu \mathrm{m}$ para $0,35 \mu \mathrm{m}$, para concentrações de $1 \%$ e $5 \%$ de argila respectivamente (Figuras 3a e 3c). Em razão do aumento da delaminação das lâminas de argila a espessura diminuiu de $0,045 \mu \mathrm{m}$ para $0,031 \mu \mathrm{m}$, e consequentemente, a razão de aspecto para o nanocompósito com $5 \%$ foi o dobro comparando com a amostra com 1\% de OMMT. Com o compatibilizante, o comprimento médio e a espessura das partículas diminuiu com o aumento da concentração de C-15A ocasionando variações pequenas na razão de aspecto em relação às amostras sem compatibilizante. 


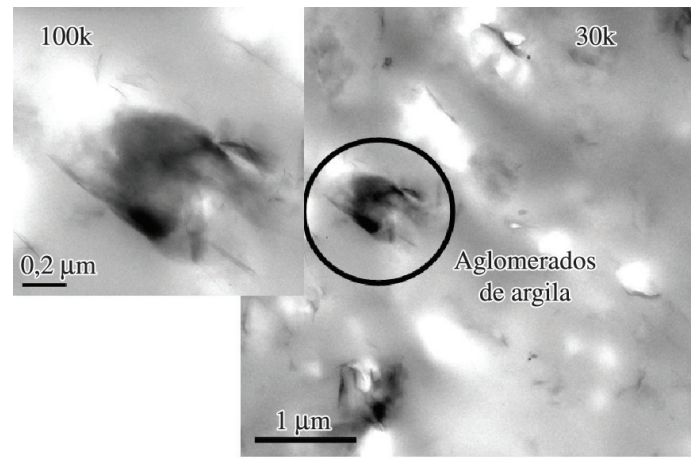

(a)

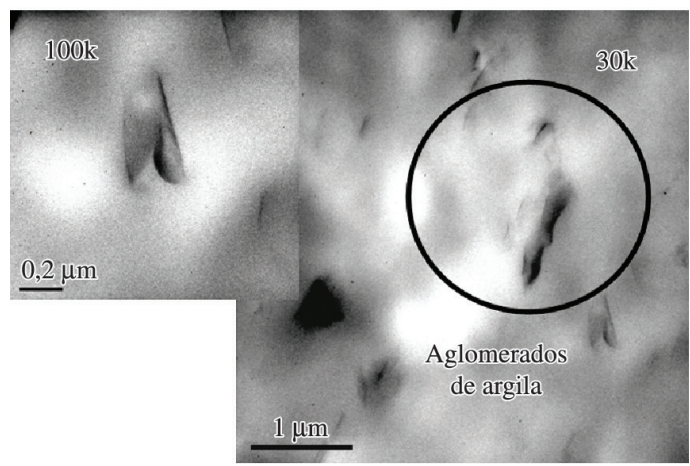

(c)

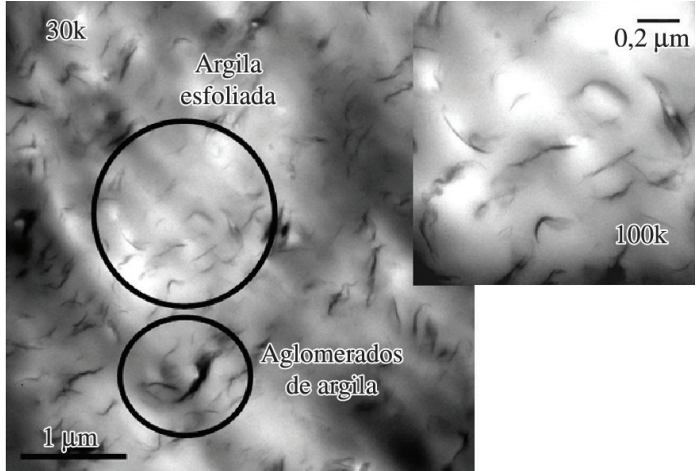

(b)

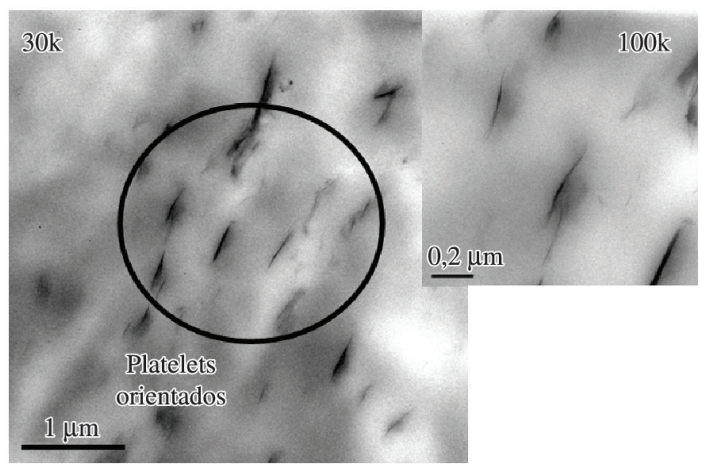

(d)

Figura 2. Micrografias de MET (a) $1 \%$ I $44 \mathrm{P}+$ PP (b) $1 \%$ I 44 + 1\% PP-g-MA + PP (c) $5 \%$ I 44 P + PP (d) $5 \%$ I 44 + 5\% PP-g-MA + PP.

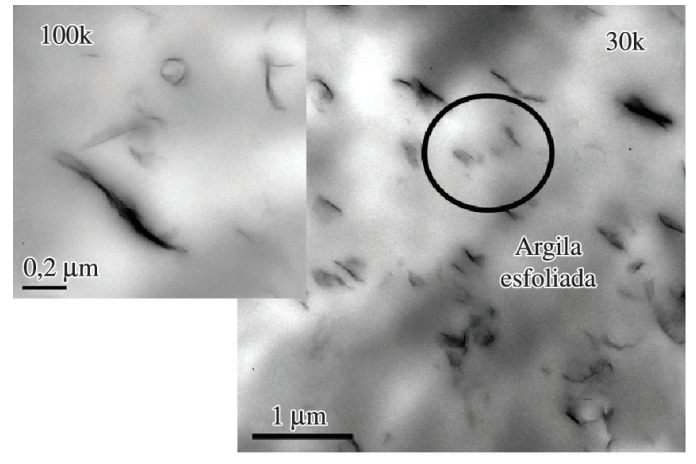

(a)

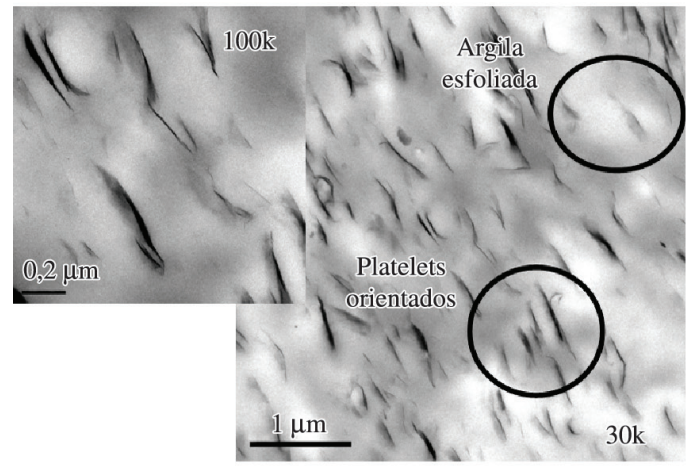

(c)

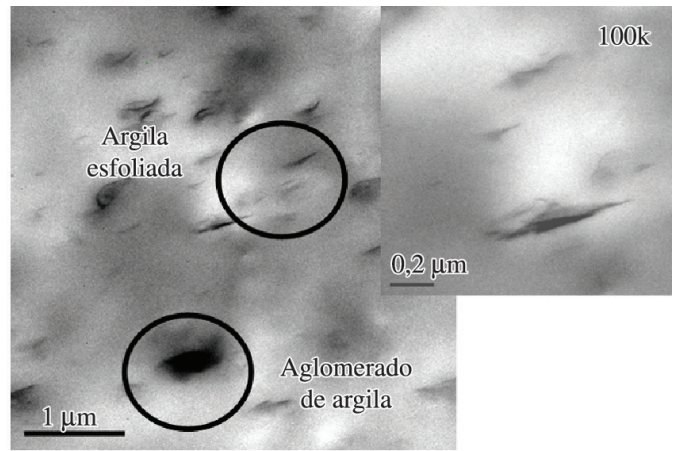

(b)

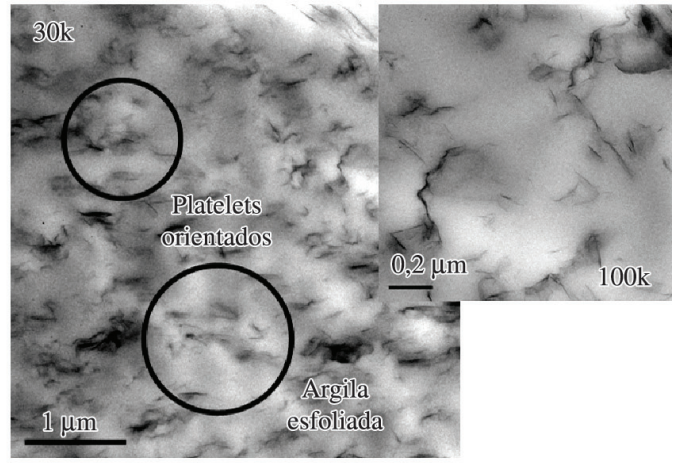

(d)

Figura 3. Micrografias de MET (a) $1 \% \mathrm{C} 15-\mathrm{A}+\mathrm{PP}$ (b) $1 \% \mathrm{C} 15-\mathrm{A}+1 \% \mathrm{PP}-\mathrm{g}-\mathrm{MA}+\mathrm{PP}$ (c) $5 \% \mathrm{C} 15-\mathrm{A}+\mathrm{PP}(\mathrm{d}) 5 \% \mathrm{C} 15-\mathrm{A}+5 \%$ PP-g-MA + PP. 
Razão de aspecto:0,35/0,031=11,2
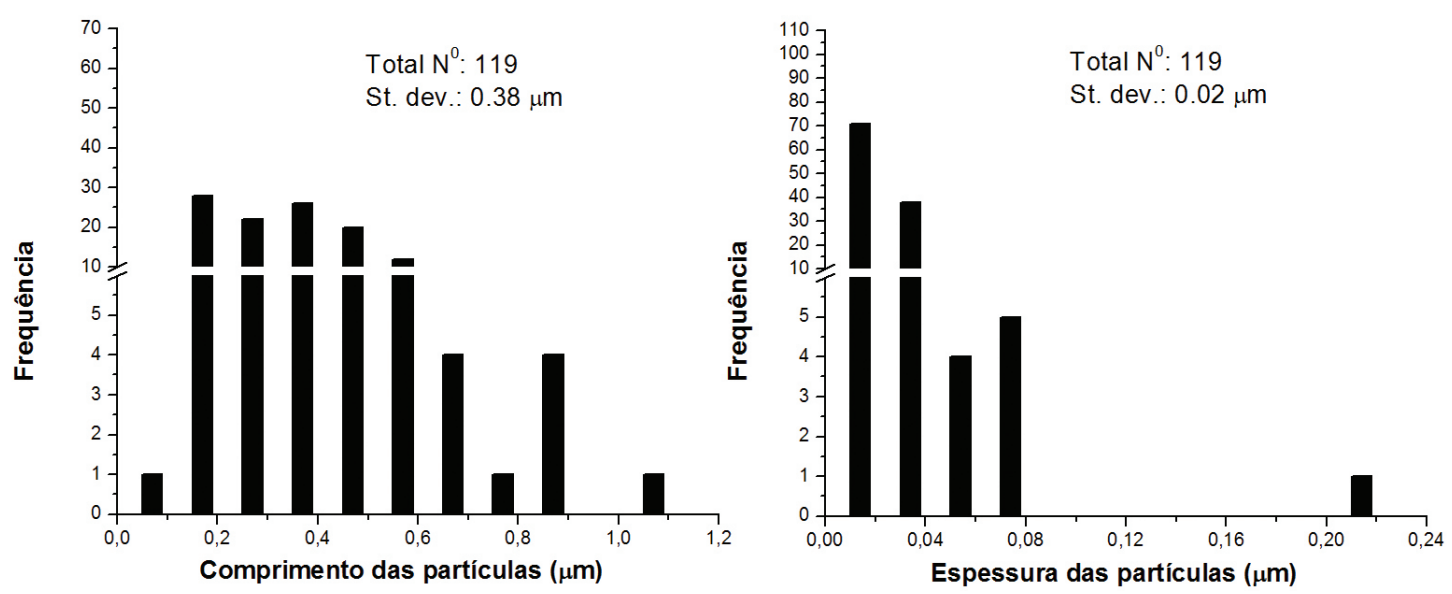

(a)

Razão de aspecto:0,21/0,021=10,0
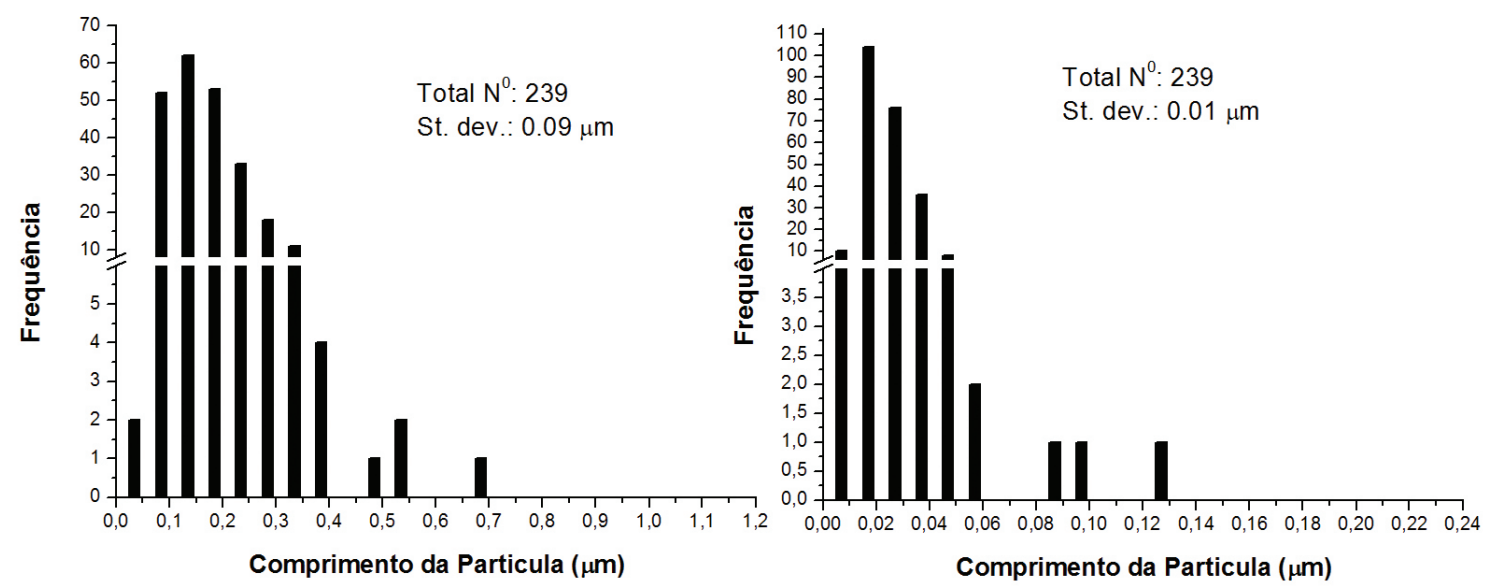

(b)

Figura 4. Histogramas do comprimento e espessura das partículas de PP/MMT: (a) 5\% C15-A + PP (b) 5\% C15-A + 5\% PP-g-MA + PP.

\section{Propriedades Mecânico-dinâmicas e Térmicas dos Nanocompósitos de PP/MMT}

O módulo de armazenamento (E') dos nanocompósitos determinado por DMA é apresentado na Tabela 3. Um comportamento similar nessa propriedade foi observado para as duas argilas. Nas amostras com $1 \%$ de argila observou-se uma leve diminuição no E' em relação ao PP puro, tendo em vista que concentrações muito pequenas de argila não conseguem atuar de forma eficiente como agente reforçante ou porque a adesão à matriz foi fraca. Na concentração de 5\% de OMMT, as partículas de argila apresentaram um efeito reforçante na matriz de PP, proporcionando um aumento no módulo de armazenamento.

A adição do agente compatibilizante não apresentou uma variação significativa no módulo de armazenamento. Como tinha sido verificado na análise morfológica, o agente compatibilizante auxiliou no aumento da esfoliação da argila, mas ao mesmo tempo se observou que as partículas não estão orientadas, provavelmente por efeito do solvente utilizado, e essa falta de orientação contribui para a diminuição da capacidade de reforço (Figuras 2c e 2d),

Na Tabela 3 também são apresentados os resultados da temperatura de fusão $\left(\mathrm{T}_{\mathrm{m}}\right)$, a temperatura de cristalização $\left(\mathrm{T}_{\mathrm{c}}\right)$ e o grau de cristalinidade $\left(\mathrm{X}_{\mathrm{c}}\right)$. Os dados mostram que a adição de argila e o uso do agente compatibilizante não modificaram significativamente a temperatura de fusão e nem grau de cristalinidade, mas a $\mathrm{T}_{\mathrm{c}}$ aumentou para as duas concentrações de argila utilizada, mostrando o efeito nucleante da nanocarga na cristalização do PP. A presença do agente compatibilizante também pode influenciar no aumento da temperatura de cristalização, visto que suas cadeias agem como uma ponte entre a superfície da OMMT e as cadeias de PP melhorando a dispersão da nanocarga na matriz ${ }^{[5]}$. Este comportamento é observado principalmente nos sistemas PP/I-44P, 
Tabela 3. Propriedades térmicas e mecânico-dinâmicas dos nanocompósitos de PP.

\begin{tabular}{lcccc}
\hline \multicolumn{1}{c}{ Amostras } & $\mathbf{E}^{\prime} \mathbf{a} \mathbf{2 3}^{\circ} \mathbf{C}(\mathbf{M P a})$ & $\mathbf{T m}^{*}\left({ }^{\circ} \mathbf{C}\right)$ & $\mathbf{T c}^{*}\left({ }^{\circ} \mathbf{C}\right)$ & $\mathbf{X c}(\boldsymbol{\%})$ \\
\hline \multicolumn{1}{c}{ PP } & $\mathbf{9 2 8}$ & $\mathbf{1 6 0}$ & $\mathbf{1 1 5}$ & $\mathbf{5 1}$ \\
\hline $1 \%$ I44P + PP & 876 & 161 & 117 & 53 \\
$1 \%$ I44P + 1\% PP-g-MA + PP & 908 & 160 & 120 & 53 \\
$5 \%$ I44P + PP & 1042 & 161 & 116 & 51 \\
$5 \%$ I44P + 5\% PP-g-MA + PP & 981 & 162 & 118 & 58 \\
$1 \%$ C-15A + PP & 885 & 160 & 118 & 55 \\
$1 \%$ C-15A + 1\% PP-g-MA + PP & 893 & 161 & 117 & 57 \\
$5 \%$ C-15A + PP & 999 & 163 & 115 & 56 \\
$5 \%$ C-15A + 5\% PP-g-MA + PP & 1009 & 163 & 115 & 55 \\
\hline
\end{tabular}

*desvio padrão: $\pm 1^{\circ} \mathrm{C}$.

em que a temperatura de cristalização aumentou para qualquer quantidade de argila.

\section{Conclusões}

O uso do solvente e o efeito da sonicação promovida pelo ultrassom, na obtenção dos nanocompósitos, promoveram o aumento da distância interlamelar de ambas as argilas. Esse processo permitiu que as cadeias poliméricas conseguissem penetrar entre essas lamelas levando a esfoliação, como mostraram as micrografias. Uma maior orientação das partículas de argila foi alcançada pelo uso da argila C-15A devido a sua maior estabilidade proporcionada pela presença de uma maior quantidade de modificador orgânico.

O agente compatibilizante também proporcionou uma melhor dispersão e distribuição das partículas. Entretanto, com relação a razão de aspecto e temperatura de cristalização, o PP-g-MA conseguiu atuar de forma mais eficiente nos nanocompósitos obtidos com a argila I44P. A rigidez dos nanocompósitos comparada ao PP puro, avaliada através do módulo de armazenamento, aumentou com o teor de argila, enquanto que o compatibilizante não apresentou nenhuma influencia significativa sobre esta propriedade.

\section{Agradecimentos}

Os autores agradecem o apoio financeiro a CAPES, CNPq e FAPERGS / PRONEX e a Braskem S.A pelos materiais fornecidos.

\section{Referências Bibliográficas}

1. Ton-That, M. T.; Perrin-Sarazin, F.; Cole,K. C.; Bureau; M. N. \& Denault, J. - Polym. Eng. Sci., 44, p.1212 (2004). http://dx.doi.org/10.1002/pen.20116

2. Santos, K. S.; Liberman, S. A.; Oviedo, M. A. S. \& Mauler, R. S. - J. Appl. Polym. Sci., 119, p.1567 (2011). http:// dx.doi.org/10.1002/app.32828

3. Silva, R. P.; Mauler, R. S.; Oliveira, R. V. B. \& Salles, C. A. - Polímeros, 20, p.46 (2010). http://dx.doi.org/10.1590/ S0104-14282010005000012

4. Bettio, P. P. S. \& Pessan, L. A. - Polímeros, 22, p.332 (2012). http://dx.doi.org/10.1590/S0104-14282012005000044

5. Santos, K. S.; Liberman, S. A.; Oviedo, M. A. S. \& Mauler, R. S.- J. Polym. Sci.: Part B: Poly. Phys., 46, p.2519 (2008). http://dx.doi.org/10.1002/polb.21604
6. Paiva, L. B.; Morales, A. R. \& Guimarães, T. R. - Polímeros, 16, p.136 (2006).

7. Dal Castel, C.; Pelegrini Jr, T.; Barbosa, R. V.; Liberman, S. A. \& Mauler, R. S.- Compos. Part A., 41, p.185 (2010). http://dx.doi.org/10.1016/j.compositesa.2009.09.017

8. Xu, W.; Liang, G.; Zhai, H.; Tang, S.; Hang, G. \& Pan, W. - Eur. Polym J., 39, p.1467 (2003). http://dx.doi. org/10.1016/S0014-3057(03)00015-6

9. Wang, Y.; Chen, F.; Li, Y. C. \& Wu, K. - Comp. Part B, 35, p.111 (2004). http://dx.doi.org/10.1016/S13598368(03)00049-0

10. Burgentzlé, D.; Duchet, J.; Gérard, J. F.; Jupin, A. \& Fillon, B. - J. Colloid Interf. Sci., 278, p.26 (2004). PMid:15313634. http://dx.doi.org/10.1016/j.jcis.2004.05.015

11. Wiewióra, A.; Pérez-Rodríguez, J. L.; Perez-Maqueda, L. A. \& Drapala, J. - Appl. Clay Sci., 24, p.51 (2003). http:// dx.doi.org/10.1016/S0169-1317(03)00133-9

12. Amash, A. \& Zugenmaier, P. - J. Appl. Polym. Sci., 63, p.1143 (1997). http://dx.doi.org/10.1002/(SICI)10974628(19970228)63:9<1143::AID-APP6>3.0.CO;2-H

13. Calcagno, C. I. W.; Mariani, C. M.; Teixeira, S. R. \& Mauler, R. S. - Polymer, 48, p.966 (2007). http://dx.doi. org/10.1016/j.polymer.2006.12.044

14. Santos, K. S.; Bischoff, E.; Liberman, S. A.; Oviedo, M. A. S. \& Mauler, R. S. - Ultrason. Sonochem, 18, p.997 (2011). PMid:21486705. http://dx.doi.org/10.1016/j. ultsonch.2011.03.011

15. Santos, K. S.; Liberman, S. A.; Oviedo, M. A. S. \& Mauler, R. S. - Compos. Part A, 40, p.1199 (2009). http://dx.doi. org/10.1016/j.compositesa.2009.05.009

16. Hotta, S. \& Paul, D. R. - Polymer, 45, p.7639 (2004). http:// dx.doi.org/10.1016/j.polymer.2004.08.059

17. Kim, D. H.; Fasulo, P. D.; Rodgers, W. R. \& Paul, D. R. - Polymer, 48, p.5960 (2007).

18. Pavlidou, S. \& Papaspyride, C. D. - Prog. Polym. Sci., 33, p.1119 (2008). http://dx.doi.org/10.1016/j. progpolymsci.2008.07.008

19. King, H. E.; Milner Jr., S. T.; Lin, Min Y.;Singh, J. P. \& Mason, T. G. - Physics Review E, 75, p.1 (2007).

20. Lee, H.; Fasulo, P. D.; Rodgers, W. R. \& Paul, D. R. - Polymer, 46, p.11673 (2005). http://dx.doi. org/10.1016/j.polymer.2005.09.068 